\title{
INCLUSÃO DIGITAL EM PORTUGAL: REFERÊNCIAS PARA A INCLUSÃO EM MOÇAMBIQUE
}

\section{DIGITAL INCLUSION IN PORTUGAL: REFERENCES FOR INCLUSION IN MOZAMBIQUE}

\author{
Delfina Lazaro Mateusa \\ Virginia Ortiz-Repisob \\ Manuel Valente Mangue
}

\begin{abstract}
RESUMO
Objetivo: O estudo analisou as políticas públicas de inclusão digital em Moçambique e em Portugal, igualmente, identificou e descreveu programas de literacia digital desenvolvidos nos dois países com o intuito de encontrar casos de boas práticas. Metodologia: A pesquisa aplicou o método qualitativo com características descritivas, o método bibliográfico e documental. Resultados: Os resultados mostram que Portugal, em comparação com Moçambique, está avançado no que se refere ao desenho e implementação de políticas públicas de inclusão digital, pois além de clarificar os eixos e o nicho da população que mais necessita ser incluída digitalmente, tem buscado, através da iniciativa Portugal InCoDe 2030, disseminar e documentar todas as estratégias de inclusão que estão a ser desenvolvidas no país. Conclusões: Dessa forma, conclui-se que Portugal tem conseguido evidenciar os aspetos superados e em atraso no que concerne ao cumprimento dos Objetivos para o Desenvolvimento Sustentável -ODS ou da Agenda Digital 2030 em relação a Moçambique, e por isso os programas desenvolvidos em Portugal podem servir como exemplos para a construção de programas de inclusão digital em Moçambique.
\end{abstract}

Descritores: Inclusão digital. ODS. Moçambique. Portugal. Políticas Públicas.

\section{INTRODUÇÃO}

O século $X X$ e os inícios do XXI estão marcados por muitas transformações, as quais influenciam os setores sociais e geram novos

\footnotetext{
a Coordenadora do curso de licenciatura em Biblioteconomia na Escola de Comunicação e Artes da Universidade Eduardo Mondlane (UEM), Moçambique. Email: 100357049@alumnos.uc3m.es

b Professora do Departamento de Biblioteconomia e Documentação da Universidade Carlos III de Madrid. Email: virginia@bib.uc3m.es

c Doutor em Ciências da Informação. Escola de Comunicação e Artes. Universidade Eduardo Mondlane (UEM), Moçambique. Email: manuel.mangue@uem.ac.mz
} 
comportamentos nos cidadãos (MALEANE, 2013), neste novo contexto, saber escrever e ler, sem dominar o uso das tecnologias de informação e comunicação-TIC, implica estar excluído da sociedade.

A falta de domínio das TIC e a exclusão acontecem porque grande parte da informação e do conhecimento está vinculada através de ferramentas tecnológicas que não estão ao alcance de muitas pessoas (ASTUDILLOTORRES; CHÉVEZ-PONCE; OVIEDO-VARGAS, 2020) devido a inúmeros fatores que podem ser: sociais, políticos, económicos, culturais, infraestruturais, etc. Então, estas questões impactam no acesso a informação e bens entre ricos e pobres dentro ou fora do mesmo país (KNOP, 2017).

O termo exclusão digital vem do inglês digital divide, que começou a ser usado na década de 1990 durante o governo de Bill Clinton nos Estados UnidosEUA (ANDRÉS; MARTíNEZ; LUGO, 2016). A autoria do termo não é clara, embora seja geralmente atribuída a Raeal Moore, pesquisador do Center for Equity Learning -ACT, ou a Lloyd Morrisset, psicólogo americano que investiga temas que relacionam a educação e as TIC.

A expressão exclusão digital é usada para referir a lacuna existente entre os conectados e os desconectados, e quando o Estado não investe na educação e nas infraestruturas capazes de reduzir as diferenças de acesso as TIC entre as zonas rurais e urbanas, raças, etnias, classes sociais e gênero (CAÑÓNRODRÍGUEZ; GRANDE DE PRADO; CANTÓN MAYO, 2016).

O conceito de exclusão digital pode ser tratado em duas etapas, que são: a conectividade e as questões sociopolíticas e econômicas (DE LA SELVA, 2015). A conectividade está relacionada com a possibilidade de se ter acesso à Internet. Nesta perspetiva, a exclusão é entendida como um problema predominantemente de acesso às tecnologias e que, portanto, pode ser resolvido pela expansão das infraestruturas; disponibilidade de equipamentos e ligações de internet (DE LA SELVA, 2015). As questões sociopolíticas e econômicas estão relacionadas com o desenvolvimento da própria sociedade do século XXI que revela grandes diferenças no uso e aplicação das ferramentas tecnológicas para o crescimento econômico, social e político (DE LA SELVA, 2015). 
Com o exposto, confirma-se que a questão da inclusão digital não tem a ver exclusivamente com o acesso às infraestruturas tecnológicas, ou seja, a garantia de que a sociedade está incluída digitalmente, não se refere somente a possibilidade de acesso a infraestrutura tecnológica, é necessário também, que esta sociedade tenha salvaguardada as condições econômicas, educacionais, culturais, políticas e psicológicas, pois a partir daí, será possível se garantir que os programas, as políticas e projetos de inclusão digital sejam eficazes.

A inclusão digital pode ser também pensada como uma política pública, na medida que se faz necessário compreender as ações ou falta de ações administrativas, técnicas e políticas do governo que por sua vez, influenciam a vida dos cidadãos.

Assim, "analisar as políticas públicas para a inclusão digital é pensar nos programas e nas políticas implementadas entre a sociedade civil e o governo de forma a se compreender como estabelecem um diálogo público e político capaz equacionar os problemas sociais relativos ao uso das TIC. Sendo que o papel do Estado e dos órgãos que executam os programas de literacia e de desenvolvimento de competências digitais, é propor iniciativas que envolvam a comunidade, entretanto com zelo pela utilização dos recursos públicos e que apresentem preocupações e ações referentes às questões sociais pertinentes para os indivíduos" (ESTEVÃO; NEVES, 2016, p. 76).

Partindo destas premissas, o presente trabalho analisou as políticas públicas de inclusão digital em Portugal e em Moçambique, em seguida, descreveu e comparou os projetos de literacia digital desenvolvidos nos países, no intuito de encontrar exemplos de boas práticas.

A identificação dos programas de literacia digital é feita a partir da localização de estratégias que estão a ser adotadas pelos países para se cumprir a Agenda 2030 e os Objetivos para o Desenvolvimento Sustentável - ODS. Estes instrumentos foram criados pela ONU e adotados a nível internacional (ONU, 2016).

A ONU está entre os autores internacionais que estabelece agendas sociais, económicas e políticas, que ao serem seguidas pelos países, podem reduzir as vulnerabilidades sociais, e neste caso, a exclusão digital. Neste sentido, os ODS foram estabelecidos pela ONU enquanto um caminho para a inclusão digital, social, política e econômica. 


\section{PROCEDIMENTOS METODOLÓGICOS}

Para o desenvolvimento deste estudo aplicou-se o método qualitativo com características descritivas. Estes métodos ajudaram na identificação e análise de políticas e projetos de inclusão digital em Portugal e em Moçambique. A pesquisa se apoiou, igualmente na revisão bibliográfica e documental sobre as políticas e programas de inclusão digital em Moçambique e em Portugal.

Devido a falta de conteúdos na internet sobre tais projetos em Moçambique, foi necessária a realização de entrevistas semiestruturadas com funcionários do Ministério da Educação e Desenvolvimento Humano e do Ministério da Ciência e Tecnologia, Ensino Superior e Técnico Profissional. As entrevistas realizadas nestas instituições ajudaram a localizar seis programas de inclusão digital em Moçambique, e destes selecionaram-se dois casos para o estudo.

A pesquisa dos programas de inclusão digital em Portugal foi feita na página web do programa: Iniciativa Nacional Competência Digitais 2030 Portugal InCoDe 2030, que é uma iniciativa integrada de políticas públicas para a inclusão digital e o desenvolvimento de competências digitais em Portugal (PORTUGAL INCODE, 2017). Neste sentido, foram identificados através desta iniciativa mais de 50 projetos e programas de inclusão digital em Portugal, dos quais foram selecionados dois casos para o presente trabalho.

\section{OS CAMINHOS PARA A INCLUSÃO DIGITAL}

A inclusão digital, enquanto um programa político e governamental, deveria diminuir as brechas e as exclusões, principalmente para os grupos marginalizados, uma vez que são estes que mais precisam das TIC para a transformação social e comunitária.

No que concerne ao conceito da inclusão digital, é necessário destacar que este termo tem sido frequentemente utilizado pelas organizações internacionais e pelo setor público, para compor um jargão atrativo capaz de garantir a solução para quase todos os entraves da sociedade contemporânea: pobreza, desigualdade social, carências educacionais, injustiça social, 
desemprego, violência, criminalidade, entre outros (BONILLA; PRETTO, 2011).

Nesta linha de ideias, o conceito de inclusão digital deve ser vinculado "a um processo que parte do acesso aos recursos digitais, mas de modo nenhum deve se restringir a ele. E para que se efetive, deve passar por um processo amplo de exercício de cidadania que abarca quatro grupos capitais: social, cultural, intelectual e técnico" (LEMOS; COSTA, 2005, p. 10).

Bonilla e Pretto (2011) destacam que existem dois tipos de inclusão digital que são: a inclusão espontânea e a inclusão induzida.

A inclusão espontânea se refere a inserção coerciva das pessoas na sociedade da informação, por exemplo, para o uso de cartões eletrônicos de débito e crédito, de smart cards em ônibus, da operação em caixas eletrônicas, do envio de imposto de renda pela internet, votação via urna eletrônica, acesso eletrônico a vários órgãos públicos, check in pela Web em viagens de avião, uso de Short Message Service - SMS em serviços de telefonia móvel, entre outros (BONILLA; PRETTO, 2011).

O conceito de inclusão espontânea remete também a cultura do consumo, típica do capitalismo, que busca estimular o consumo desenfreado a partir da criação de ferramentas e aplicações tecnológicos capazes de seduzir aos consumidores e novos usuários (SGORLA; LINDINO, 2015), mesmo quando estes não tem competências e conhecimentos suficientes para maneja-los.

Por outro lado, a inclusão induzida que é considerada efetivamente como inclusão digital pelos autores, tem a ver com o resultado de um trabalho educativo e de políticas públicas que permitem que os grupos excluídos aproveitem as TIC para o seu desenvolvimento social e pessoal (BONILLA; PRETTO, 2011). A inclusão induzida tem como base três dimensões que são: a técnica, cognitiva e econômica.

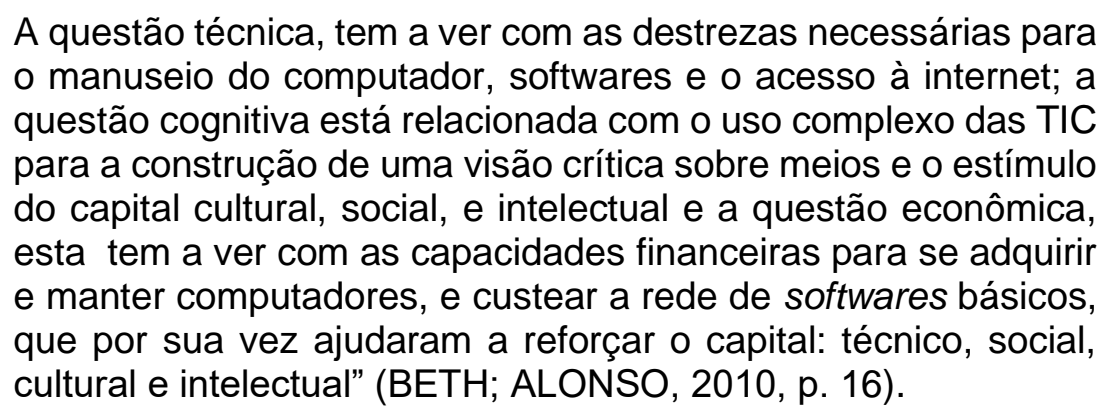

Ao se desenvolver a inclusão na perspectiva induzida se espera que os cidadãos 
alcancem a Literacia digital que é entendida como um conjunto de competências necessárias para que, além da leitura e da escrita por meio das TIC, os cidadãos possam, ao mesmo tempo, compreender o uso das TIC, buscar, selecionar e analisar as informações que circulam neste meio, seja para produzir novas informações ou conhecimento (OLIVEIRA, 2015).

Tratando-se de um estudo sobre inclusão digital numa perspectiva de diminuição das brechas digitais para se "garantir a igualdade social entre as pessoas" (ONU, 2016, p. 10), presume-se que seja necessário que os programas de inclusão digital sejam pensados na perspectiva da inclusão induzida, pois esta vertente tende a destacar a necessidade de existirem políticas, programas, estratégias e ações necessárias para o desenvolvimento de competências informacionais e digitais.

Para avaliar os programas de inclusão digital Brandão (2009) apresenta o Indicador de Alfabetização Digital e Social - IADS que é um instrumento para avaliação de habilidades no processo da alfabetização digital e social dos indivíduos. Este indicador, compreende as seguintes dimensões:

a) Uso pessoal e lazer: para representar as habilidades de uso pessoal das TIC em sua aplicação cotidiana e para o lazer;

b) Uso autônomo: para representar as habilidades de uso das TIC no trabalho, de maneira autônoma, para a resolução de problemas, geração e produção de informações e conhecimentos;

c) Uso social e colaborativo: para representar as competências e uso das TIC para o relacionamento em redes sociais, comunidades virtuais, aprendizagem social, participação e colaboração em processos de construção e produção colaborativa para o benefício social e institucional;

d) Uso inovador e sustentável: para representar as competências de uso criativo e inovador das TIC na solução de problemas comunitários preservando-se os valores, identidades, cultura e recursos ambientais (BRANDÃO, 2009).

Neste caso, para a identificação dos projetos de inclusão digital, se tem como referência o indicador IADS e as questões de: idade, raça, gênero, localização geografia (zona urbana ou rural) e poder económico, uma vez que 
são relatadas como importantes para a implementação dos ODS (ONU, 2016).

\subsection{Políticas Públicas de Inclusão Digital em MoçambiQue}

As iniciativas de inclusão digital enquanto política pública em Moçambique, foram anunciadas quando se aprovou no ano de 2000 a política de informática pela Resolução no 12/2000. Esta política preconiza dar acesso universal à informação para melhorar o desempenho político-social e profissional dos cidadãos; melhorar a eficiência dos sectores públicos e privado e promover o investimento em TIC; contribuir para redução das assimetrias regionais, entre as zonas urbanas e rurais em diferentes segmentos da sociedade, promovendo a igualdade de acesso às oportunidades de desenvolvimento (MOÇAMBIQUE, 2000).

Apesar de Moçambique ter privilegiado a informação como um recurso importante para a transformação, não foram observados na Política de Informática os aspetos básicos inerentes aos estudos de viabilidade e o nível de interesse e adequação dos cidadãos às TIC (DIAS; PINHEIRO, 2012).

Após esta experiência e as tentativas de implementação do plano estratégico da Política de Informática, bem como da Política para o Governo Eletrônico, derivados da Política de Informática, o ano de 2016 foi marcado no âmbito da inclusão digital pela revogação da Lei no 8/2004, referente às telecomunicações, e a aprovação da Lei nํㄴ 4/2016. Esta última, tinha como um dos objetivos: promover o acesso e o serviço universal às telecomunicações; promover investimentos em infraestruturas e a partilhar as infraestruturas existentes para a promoção da inovação e desenvolvimento tecnológico (MOÇAMBIQUE, 2016).

Com vista a garantir-se o acesso universal às telecomunicações, tendo como base a lei das telecomunicações, foi aprovado no ano de 2017, a estratégia nacional de banda larga, com o objetivo de fomentar e difundir o uso e o fornecimento de bens e serviços através das TIC; massificar a cobertura dos serviços básicos de banda larga para as zonas rurais e melhorar a disponibilidade de ligações de banda larga através da fibra ótica para as zonas urbanas (MINISTÉRIO DOS TRANSPORTES E COMUNICAÇÃO, 2017).

Com isso, a Estratégia Nacional de Banda Larga preconiza: 
a) Acelerar o desenvolvimento econômico e social;

b) Promover a inclusão digital;

c) Reduzir as desigualdades sociais e regionais;

d) Promover a geração de emprego e aumento da renda;

e) Ampliar os serviços do Governo Eletrônico e facilitar aos cidadãos o uso dos serviços do Estado; e

f) Promover a capacitação da população para o uso das tecnologias de informação e comunicação (MINISTÉRIO DOS TRANSPORTES COMUNICAÇÃO, 2017).

Apesar dos objetivos terem sido muito bem definidos na Lei das Telecomunicações e na Estratégia Nacional de Banda Larga, o processo de implementação parece estar longe de ser alcançado, pois o cenário de Moçambique frente a estas questões ainda deixa a desejar, porque os serviços de banda larga continuam a cobrir de forma prática, apenas as áreas urbanas, enquanto que uma percentagem significativa da população vive em áreas rurais (MINISTÉRIO DOS TRANSPORTES COMUNICAÇÃO, 2017), condicionando assim, o acesso às inúmeras vantagens que estes serviços podem oferecer à sociedade.

A afirmação anterior é apoiada por Herrmann (2019) quando mostra que os últimos estudos do Banco Mundial sobre o uso de TIC na África Subsaariana (onde Moçambique faz parte), indicam que apenas uma em cada cinco pessoas usa a Internet. Ademais, os grandes e importantes atores da infraestrutura da Internet na África são as empresas multinacionais de telecomunicações, e não empresas estatais, o que de certa forma, impossibilita o acesso massivo às tecnologias e a inclusão digital.

Sobre esta questão, é necessário também destacar que o acesso às infraestruturas, banda larga e internet, em África, e precisamente em Moçambique, não garantiriam em si à inclusão digital pois mais do que existirem esses aspetos, é importante que se garanta a inclusão social, económica, cultural e política. E esta última, deve dar maior ênfase à educação, por ela ser capaz de garantir a construção de uma cidadania criativa e empoderada.

Ainda sobre as políticas de inclusão digital em Moçambique, o ano de 2018 foi marcado pela aprovação da Política para a Sociedade da Informação pela 
resolução ํo 17/2018. Esta política tem como propósito: estabelecer linhas orientadoras para o desenvolvimento sustentável, visando tornar Moçambique numa sociedade inclusiva e competitiva através da massificação das TIC. A política estabelece como objetivos específicos, referentes à inclusão digital, as seguintes:

a) Assegurar o desenvolvimento do capital humano qualificado e com competências necessárias para responder aos desafios da modernização da sociedade;

b) Garantir a existência de infraestruturas inclusivas que promovam 0 acesso universal às TIC; e

c) Sensibilizar os vários atores da sociedade para a importância das TIC, enquanto eixo de desenvolvimento económico e social (MOÇAMBIQUE, 2018).

De modo geral, esta é a primeira política moçambicana que aponta de forma clara e incisiva os elementos e os conceitos tratados nos ODS referente a inclusão digital. Por exemplo, ao tratar-se dos princípios da infoinclusão, se destaca neste instrumento, que as TIC devem ser um bem disponível para toda a população independentemente da sua localização geográfica, idade, género e classe social, pois somente assim se poderá reforçar e garantir a literacia digital. Além disso, se descreveu o papel das TIC para a melhoria dos sectores da educação, agricultura, economia, saúde, governação e desenvolvimento urbano no país.

A anunciação dos elementos e conceitos relativos aos ODS podem ser tidos como positivos, uma vez que o país demonstra a partir deste instrumento legal o seu compromisso com os ODS, porém vale mencionar que a implementação e o alcance dos ODS partem da criação de sinergias entre os programas e políticas que buscam reduzir as exclusões sociais, educacionais, econômicas, de emprego, saúde etc.

Isto porque, grande parte dos programas de inclusão digital em Moçambique não respondem às necessidades específicas das comunidades que muitas vezes possuem necessidades que não são necessariamente tecnológicas, tais como: a falta de eletricidade, de escolas em condições, de água potável e de hospitais apetrechados (KARBERG, 2015).

Não menos importante, é preciso destacar que $45 \%$ da população do país 
é analfabeta, e este problema, aliado ao facto de existirem telecentros exíguos e precários, dificulta a implementação de programas de inclusão digital.

\subsection{Políticas Públicas de Inclusão Digital em Portugal}

As políticas públicas para a inclusão digital em Portugal são orientadas pela Iniciativa: Agenda Digital para a Europa. Esta agenda foi lançada em maio de 2010, com o objetivo de contribuir para a inclusão digital e a literacia digital da população europeia (PARLAMENTO EUROPEU, 2014).

Assim, em 2015, Portugal lançou a Estratégia Nacional de Inclusão e Literacia Digital para o período de 2015-2020, com o objetivo de fomentar a participação e o comprometimento da sociedade através da apropriação extensiva e sistemática das TIC por idosos, adultos com baixos níveis de escolaridade e pessoas inativas com baixas qualificações profissionais (FUNDAÇÃO PARA A CIÊNCIA E A TECNOLOGIA, 2015).

Esta política estabelece como objetivos estratégicos os seguintes:

a) Reduzir a percentagem de não utilizadores da Internet;

b) Mobilizar uma infraestrutura operacional que permita formação presencial: espaços, equipamentos e formadores;

c) Incentivar o desenvolvimento de materiais pedagógicos para (auto)formação;

d) Aumentar as competências digitais da população portuguesa;

e) Criar uma rede de intervenção multistakeholder (a Rede TIC e Sociedade); e

f) Incentivar a melhoria da interface entre os serviços online e os cidadãos (acessibilidade e usabilidade) (FUNDAÇÃO PARA A CIÊNCIA E A TECNOLOGIA, 2015).

No que concerne aos aspetos de infraestrutura, Portugal apresenta situações favoráveis, pois se verifica que a nível dos países da União Europeia, se encontra numa boa posição no que refere à capacidade de conectividade e a extensão da Banda Larga.

No entanto, é preciso destacar que no que toca às questões de competências e habilidades para a utilização da internet por parte da população, ainda há muito que se fazer, pois ao se comparar o desempenho do país com 
outros países da União Europeia, se verifica que a população portuguesa tem falta de competências, e esta situação dificulta o processo de acesso à informação e a serviços que demandam um pouco mais de habilidades tecnológicas, como: as compras online e o acesso aos serviços bancários (FUNDAÇÃO PARA A CIÊNCIA E A TECNOLOGIA, 2015).

Para superar estes desafios, o Governo português anunciou em abril de 2017 o programa Portugal InCoDe 2030, com o objetivo de promover projetos de literacia digital, para crianças, mulheres, pessoas empregados $\mathrm{e}$ desempregados, estudantes, adultos etc., como forma de garantir que todos desenvolvam competências digitais em diferentes níveis de profundidade e proficiência (FERREIRA, 2018),

Este programa tem como objetivo, garantir a literacia digital e a inclusão digital para o exercício da cidadania nos seguintes eixos, que são:

a) Inclusão: Assegurar o acesso equitativo às tecnologias digitais a toda a população, para obtenção de informação, comunicação e interação.

b) Educação: Assegurar a educação das camadas mais jovens da população através do estímulo e esforço nos domínios da literacia digital e das competências digitais em todos os ciclos de ensino e de aprendizagem ao longo da vida.

c) Qualificação: Capacitar profissionalmente a população ativa dotando-a de conhecimentos necessários à integração num mercado de trabalho que depende fortemente de competências digitais.

d) Especialização: Promover a especialização em tecnologias digitais e aplicações para a qualificação do emprego e a criação de maior valor acrescentado na economia.

e) Investigação: Garantir as condições para a produção de novos conhecimentos e a participação ativa em redes e programas internacionais de Investigação e Desenvolvimento - I\&D (PORTUGAL INCODE).

Assim, no âmbito deste programa, são desenvolvidos projetos de inclusão digital para a população dentro dos eixos descritos. Ainda nesta linha, o Governo português aprovou através da Resolução no 30/2020 o Plano de Ação Para a Transição digital em Portugal. Esta estratégia preconiza, igualmente, capacitar e 
incluir digitalmente as pessoas, garantir a transformação digital nas empresas e digitalizar o Estado português (REPÚBLICA DE PORTUGAL, 2020).

\subsection{Projetos de Inclusão Digital em Portugal e Moçambique}

\subsubsection{Casos de Portugal}

a) Projeto: Lídia, Literacia digital de Adultos4: É um projeto europeu dedicado à criação de materiais destinados a professores e educadores nas diferentes áreas curriculares e níveis de escolaridade. O projeto está a ser implementado em 5 países: Portugal, Espanha, Holanda, Itália e Grécia. As atividades para a formação foram desenhadas de acordo com os interesses do público-alvo, este grupo foi composto por adultos refugiados em Portugal. As atividades foram desenhadas tendo em conta os seguintes pontos:

- Informação: Agrupa interesses relacionados com o acesso à informação disponível na internet;

- Comunicação: Considera os interesses relacionados com objetivos de comunicação através da internet e outros canais;

- Produção: Relacionados aos interesses para a criação de algo, ou de conteúdos;

- Lazer: Agrupa atividades de lazer para adultos e com carácter lúdico;

- Dia-a-dia: Interesses decorrentes da necessidade de resolver problemas concretos do cotidiano.

b) Projeto: LIFT- Ladies Code Their Future5: pertence aos países membros da União Europeia e é cofinanciado pelo European Region Action Scheme for the Mobility of University Students - ERASMUS+. O projeto busca aumentar as competências digitais das mulheres em situação de desfavorecimento social: desempregadas, a viver nas

\footnotetext{
${ }^{4}$ Dispoível em: http://lidia.ie.ulisboa.pt

${ }^{5}$ Disponível em: https://ladiescode.eu/en/learning-platform/register
} 
zonas rurais, com baixo nível educacional, e não enquadradas em sistemas de processos de ensino regular. Tem como objetivos principais: aumentar a confiança das mulheres nas suas capacidades através de um programa de formação e mentoria; aumentar a literacia digital e competências básicas em programação; reforçar competências transversais como a criatividade, a cooperação interpessoal, a cooperação intercultural, a comunicação e a orientação para a resolução de problemas.

\subsubsection{Casos de Moçambique}

a) KOREA Funds-in-Trust ICT, Transforming Education in Africa - KFIT: o projeto foi criado com o objetivo de aproveitar o potencial das tecnologias de informação e comunicação para potencializar e garantir a equidade no sector da educação em Moçambique, Ruanda e Zimbabwe. O projeto foi financiado pelo governo sul-coreano e implementado em Moçambique em 2018. Participaram deste projeto professores, alunos de escolas públicas primárias e secundárias; instituições de ensino superior; legisladores e administradores educacionais. O projeto pretendia para Moçambique: estabelecer um modelo de escola eletrônica no ensino básico e secundário, de forma que os alunos pudessem se familiarizar com as competências do século XXI, tais como: melhoria da comunicação a partir das TIC e aquisição de habilidades para a aprendizagem à distância.

b) Projeto Africa Code Week: é um projeto anual, que realizou até então 12 edições em Moçambique. Tem como objetivo, formar jovens africanos em competências tecnológicas para a vida. Os conteúdos programados estão relacionados com a codificação informática. $O$ projeto é também responsável pela formação de educadores que, posteriormente, são responsáveis pela formação dos alunos.

\section{ANÁLISE E DISCUSSÃO}

Partindo dos dados apresentados, se percebe que para além de Portugal 
estar comprometido em garantir o cumprimento da Agenda 2030 e dos ODS, a partir da definição de políticas e planos de ação para a concretização das estratégias de inclusão e de literacia digital, tem agrupado, através de uma plataforma: InCoDe 2030, os programas e as estratégias desenvolvidas para garantir a inclusão dentro dos aspetos que precisam ser trabalhados no país para se garantir a inclusão digital dos indivíduos.

Em Moçambique, mesmo existindo uma Agenda Digital, não foi possível identificar-se planos de ações concretos para a inclusão e literacia digital, o processo de implementação das políticas e das estratégias aprovadas para a inclusão, não apresentam planos de ações ou programas de literacia digital que foram desenvolvidos neste âmbito.

Apesar de a Política de informática, a Lei das Telecomunicações, a Estratégia Nacional de Banda Larga e a Política para a Sociedade de Informação trazerem procedimentos que podem orientar a inclusão digital e a literacia digital, e esta última política, fazer menção ao conceito de literacia digital para o desenvolvimento sustentável, precisa-se considerar que estes dispositivos legais talvez sejam instrumentos capazes de estimular o desenho de uma política pública para a literacia e inclusão digital, uma vez que, ainda é necessário definirse ações e programas de inclusão que se espelhem nas necessidades e características dos grupos sociais.

A falta de uma política especifica de literacia e inclusão digital em Moçambique dificultou a identificação de estratégias e ações iguais as encontradas em Portugal, também, percebeu-se não existir clareza nas estratégias e ações desenvolvidas para garantir a literacia e inclusão digital das pessoas, principalmente as mais vulneráveis.

No que refere às ações para a inclusão digital, a iniciativa Portugal InCoDe 2030 pode ser citada como um exemplo de boa prática, porque através dela, Portugal mostra seu compromisso nacional de disseminar estratégias e programas específicos de inclusão digital para a sociedade. Além disso, mostra através desta iniciativa as linhas, os conteúdos, os programas e os grupos sociais envolvidos no processo de inclusão.

No que refere aos estudos de caso, se pode constatar que os projetos 
descritos dos dois países, se encaixam dentro das dimensões de inclusão digital descritas por Brandão (2009) e se enquadram dentro do conceito de inclusão digital induzida.

Outrossim, os estudos de caso dos dois países, buscaram de forma específica ou abrangente desenvolver conteúdos formativos que pudessem garantir a inserção dos cidadãos, como mulheres; alunos; professores; adultos; refugiados, etc., em dimensões como: lazer; autonomia; uso social, colaboração e utilização inovadora e sustentável das TIC. Porém, de todos eles, o projeto Lidia: literatura digital para adultos, é identificado como o programa que buscou explorar quase todas as dimensões de inclusão digital mencionadas por Brandão.

Outra questão que foi verificada no projeto Lidia, é que as atividades e os conteúdos programados para a formação foram desenhados tendo em conta as necessidades dos adultos refugiados, e isto, faz considerar a necessidade e a importância de se desenhar projetos de inclusão digital a partir das características e necessidades formativas e informativas dos formandos, porque a partir disto, é possível se garantir que os conteúdos programados para a formação se ajustem ao perfil do grupo que precisa ser incluído digitalmente.

No caso de Moçambique, apesar da abrangência do projeto KFIT em termos do público abrangido (alunos, professores, servidores públicos, etc.), se observa que apenas foram explorados conteúdos referentes ao desenvolvimento de competências digitais para a educação formal.

Esta situação pode talvez estar relacionada com o fato de muitas estratégias moçambicanas dependerem de financiamento externo, limitando, desta forma, as possibilidades de desenho e realização de iniciativas com conteúdos diversificados e adequados às características do público-alvo, bem como, adequar estes conteúdos aos indicadores para o desenvolvimento sustentável, como: sexo, idade, localização geográfica e situação econômica.

Tendo em conta as dificuldades enfrentadas por Moçambique na implementação de projetos e políticas de inclusão digital, os exemplos e as experiências encontradas em Portugal, com destaque ao InCoDe 2030, mostram que Moçambique poderia, também, investir na criação de uma plataforma digital que acopla todos os programas e estratégias de inclusão digital. $\mathrm{O}$ desenho de esta 
plataforma poderia ajudar a definir domínios ou dimensões da inclusão digital em Moçambique, facilitaria a definição de prioridades no processo da inclusão. Ademais, contribuiria para a identificação de parceiros que pudessem ajudar a implementar as estratégias a partir do interesse das instituições públicas e dos próprios parceiros.

\section{CONSIDERAÇÕES FINAIS}

A identificação de programas de literacia digital em Portugal e Moçambique ajudou a perceber o nível de comprometimento dos países para o cumprimento dos ODS e para a inclusão digital.

As políticas públicas aprovadas em cada país, mostram que, não basta apenas se criar instrumentos legais para que se garanta a inclusão digital de todos, pois é necessário na mesma medida, que se desenhem planos e programas de ação, semelhantes aos encontrados em Portugal.

As experiências de inclusão digital identificadas em Portugal, chamam para a necessidade de Moçambique documentar e categorizar as estratégias e projetos de literacia digital de acordo com o público-alvo, assunto, idade e as questões sociais que se pretendem alcançar através de formações, workshops, seminários etc.

Em suma, se considera que os objetivos desenhados para o estudo foram alcançados, pois os programas e projetos de inclusão digital descritos, são capazes de servir de referência para a criação e definição de metodologias práticas para a inclusão digital em Moçambique. No entanto, vale realçar que as iniciativas portuguesas deveriam servir de inspiração para Moçambique e não necessariamente ser copiadas, uma vez que, apesar de existirem algumas semelhanças no que refere ao público que se pretende incluir digitalmente, a realidade social, de recursos humanos e de infraestruturas, econômica e cultural é diferente.

\section{REFERÊNCIAS}

ANDRÉS, Ana María Márquez; MARTíNEZ, Jorge Antonio Acevedo; LUGO, David Castro. Brecha digital y desigualdad social en México. Economía

Coyuntural, Santa Cruz, v. 1, n. 2, p. 89-136, abr./jun. 2016.

ASTUDILLO-TORRES, Martha Patricia; CHÉVEZ-PONCE, Florlenis; OVIEDOVARGAS, Yesenia. La exclusión social y las tecnológias de la información y la 
comunicación: Una visiión estadística de su relación en la educación superior. LiminaR. Estudios Sociales y Humanísticos, v. 18, n. 1, p. 177-193. 2020.

BETH, Luiza; ALONSO, Nunes. Inclusão digital e inclusão social: contribuições e metodologias. Barbarói, Santa Cruz do Sul, n. 32, p. 154-177, jan./jul. 2010.

BONILLA, Maria Helena Silveira; PRETTO, Nelson De Luca. (org.). Inclusão polêmica contemporânea. Salvador: EDUFBA, 2011.

BRANDÃO, Maria de Fátima. Um Modelo de Avaliação de Programa de Inclusão Digital e Social. 2009. 138 f. Tese (Doutorado em Psicologia Social) - Universidade de Brasília, Brasília, 2009.

CAÑÓN-RODRÍGUEZ, Ruth; GRANDE DE PRADO, Mario; CANTÓN MAYO, Isabel. Brecha Digital: impacto en el desarrollo social y personal-factores asociados. Tendencias pedagógicas, Madrid, n. 28, p. 115-132, 2016.

DE LA SELVA, Alva Alma Rosa. Los nuevos rostros de la desigualdad en el siglo XXI: la brecha digital. Revista mexicana de Ciencias Políticas y Sociales, Ciudad de México, v. 60, n. 223, p. 265-285, jan./abr. 2015.

DIAS, Adélio; PINHEIRO, Marta Macedo. Política de Governo Eletrônico em Moçambique: um olhar na perspectiva de uma cultura de informação. Informação \& Informação, Londrina, v. 17, n. 1, p. 60-77, jan./jun. 2012. Disponível em: http://www.uel.br/revistas/uel/index.php/informacao/article/view/10292. Acesso em: 22 mar. 2021.

ESTEVÃO, Pricila; NEVES, Diego. Políticas públicas de inclusão digital no meio rural : um estudo sobre os Territórios Digitais. Revista Desenvolvimento Social, Montes Claros, v. 1, n. 17, p. 75-89, 2016.

FERREIRA, Sandra Luísa Oliveira do Nascimento. Formação no domínio da literacia digital em contexto de trabalho. 2018. 83 f. Dissertação (Mestrado em Educação) - Universidade de Lisboa, Lisboa, 2018.

FUNDAÇÃO PARA A CIÊNCIA E A TECNOLOGIA. Estratégia Nacional para a Inclusão e Literacia Digital 2015-2020, 2015. Disponível em: https://www.ticsociedade.pt/docs/ENILD.pdf. Acesso em: 10 de fev. de 20121.

KARBERG, Sindy. Participação Política das Mulheres e a sua influência para uma maior Capacitação da Mulher em Moçambique. Maputo, 2015. Disponível em: http://library.fes.de/pdf-files/bueros/mosambik/13026.pdf. Acesso em: 01 jan. 2020.

KNOP, Manuel Ferreira Trezza. Exclusão digital, diferenças no acesso e uso de tecnologias informação e comunicação: questões conceituais, metodologias e empíricas. Caderno Eletrônico de Ciências Sociais, Vitória, v. 5, n. 2, p. 3859. 2017. 
LEMOS A.; COSTA, L. F. Um modelo de inclusão digital: o caso da cidade de Salvador. Revista de Economía Política de las Tecnologías de la Información y Comunicación. v. 7, n. 3, 2005.

MALEANE, Susana Otília Tomás. Tecnologias de informação e comunicação como um meio de inclusão e exclusão social em Moçambique: o caso do ensino superior. Revista Ibero-Americana de Ciência da Informação, Brasília, v. 9, n. 1, p. 308-309, 2013.

MOÇAMBIQUE. Ministério dos Transportes e Comunicações. Proposta de resolução que aprova a Estratégia Nacional de Banda Larga. Ministério dos Transportes e Comunicações: Maputo, 2017.

MOÇAMBIQUE. Lei ํo 4/2016 de 3 de junho de 2016. Institui a lei das Telecomunicações. Assembleia da República: Maputo. 2016.

MOÇAMBIQUE. Resolução 17/2018 de 21 de junho de 2018. Institui a Política para Sociedade de Informação. Assembleia da República: Maputo. 2018.

MOÇAMBIQUE. Resolução no 28/2000 de 18 de dezembro de 2000. Institui a Política de Informática. Assembleia da República: Maputo.2000.

OLIVEIRA, Michele Mezari. Revisão de literatura: Literacia Digital. In: CONGRESSO NACIONAL DE EDUCAÇÃO, 12., 2015. Anais [...]. Paraná: [s. n.], 2015. p. 14348-14356.

ORGANIZAÇÃO DAS NAÇÕES UNIDAS (ONU). Marco de indicadores mundiales para los objetivos de desarrollo sostenible y metas de la agenda 2030 para el desarrollo sostenible. Labor de la Comisión de Estadística en relación con la Agenda 2030 para el Desarrollo Sostenible, 2016. Disponível em:

https://unstats.un.org/sdgs/indicators/Global\%20Indicator\%20Framework\%20aft er\%202020\%20review_Spa.pdf. Acesso em: 21 jun. 2021.

PARLAMENTO EUROPEU. Uma agenda digital para a europa. Luxemburgo, 2014. Disponível em: https://op.europa.eu/en/publication-detail//publication/27a0545e-03bf-425f-8b09-7cef6f0870af/language-pt. Acesso em: 21 jun. 2021.

PORTUGAL INCODE. Portugal InCoDe.2030, 2017. Disponível em: https://www.InCoDe2030.gov.pt/InCoDe2030. Acesso em: 21 jun. 2021.

REPÚBLICA DE PORTUGAL. Plano de ação para a transição digital de Portugal. Economia e Transição Digital. Lisboa, 2020. Disponível em: https://www.portugal.gov.pt/gc22/portugal-digital/plano-de-acao-para-atransicao-digital-pdf.aspx. Acesso em: 21 jun. 2021.

SGORLA, Kristian; LINDINO, Terezinha Corrêa. Capitalismo, sociedade de consumo e seus reflexos na educação contemporânea. Pleiade, Foz do 
Iguaçu, v. 9, n. 18, p. 42-51, 2015.DIGITAL INCLUSION IN PORTUGAL: REFERENCES FOR INCLUSION IN MOZAMBIQUE

\begin{abstract}
Objective: The study analyzed the public policies for digital inclusion in Mozambique and Portugal and as well identified cases of good practices in inclusion projects or programs developed in both countries. Methodology: The research applied the qualitative method with descriptive characteristic as well as the bibliographic and documental method. Results: The findings show that Portugal, compared to Mozambique is advanced in terms of the design and implementation of public policies for digital inclusion, as besides clarifying the axes and niche of the population that most needs to be digitally included, it has sought through the Portugal InCoDe 2030 initiative to disseminate and document all the inclusion strategies that are being developed at country level. Conclusions: Thus, it is concluded that Portugal has managed to highlight the overcome and overdue aspects regarding the fulfillment of the Sustainable Development Goals - SDGs or the 2030 Digital Agenda in relation to Mozambique, and therefore the programs developed in Portugal can serve as good examples and practices for the construction of digital inclusion programs in Mozambique.
\end{abstract}

Descriptors: Digital inclusion. ODS. Mozambique. Portugal. Policies Public.

\title{
INCLUSIÓN DIGITAL EN PORTUGAL: REFERENCIAS PARA LA INCLUSIÓN EN MOZAMBIQUE
}

\begin{abstract}
RESUMEN
Objetivo: El estudio analizó las políticas públicas para la inclusión digital en Mozambique y Portugal, y también identificó casos de buenas prácticas en políticas y proyectos de inclusión desarrollados en ambos países. Metodología: La investigación aplicó el método cualitativo con características descriptivas, además, se ha basado en el método bibliográfico y documental. Resultados: Los resultados muestran que Portugal, en comparación con Mozambique, está avanzado en cuanto al diseño e implementación de políticas públicas de inclusión digital, ya que además de aclarar los ejes y nicho de la población que más necesita ser incluida digitalmente, ha buscado, a través de la iniciativa Portugal InCoDe 2030 difundir y documentar todas las estrategias de inclusión que se están desarrollando en el país. Conclusiones: Así, se concluye que Portugal ha logrado resaltar los aspectos superados y atrasados en cuanto al cumplimiento de los Objetivos de Desarrollo Sostenible - ODS o la Agenda Digital 2030 en relación con Mozambique, y por lo tanto los programas desarrollados en Portugal pueden servir como ejemplos de buenas prácticas en la construcción de programas de inclusión digital en Mozambique.
\end{abstract}

Descriptores: Inclusión digital. ODS. Mozambique. Portugal. Políticas publicas.

Recebido em: 15.10 .2021

Aceito em: 18.11.2021 